Recepción: 07 / 11 / 2016

Aceptación: 15 / 02 / 2017

Publicación: 18 / 05 / 2017

Ciencias económicas y empresariales

Artículo de investigación

\title{
Medición de la rentabilidad para los accionistas. ¿Es el ROE un indicador confiable para evidenciar la rentabilidad de los accionistas?
}

Measurement of profitability for shareholders. Is the ROE a reliable indicator to show the profitability of shareholders?

Medir o retorno aos acionistas. É um indicador confiável ROE para demonstrar a rentabilidade dos acionistas?

\author{
Luis M. Bejar-León ${ }^{1}$ \\ luis.bejarl@ug.edu.ec \\ Elaine R. Jijón-Gordillo " \\ elainejijon@hotmail.com
}

Correspondencia: luis.bejarl@ug.edu.ec

\footnotetext{
${ }^{\text {I }}$ Magister en Negocios Internacionales y Gestión de Comercio Exterior, Diploma Superior en Economía Internacional, Diploma Superior en Pedagogía Universitaria, Ingeniero Comercial, Docente de la Universidad de Guayaquil, Guayaquil, Ecuador.

II Magister en Administración de Empresas, Magister en Administración de Negocios, Economista, Docente de la Universidad de Guayaquil, Guayaquil, Ecuador.
} 



\section{Resumen}

Es de mucho interés conocer la rentabilidad que tenemos al invertir nuestro capital en una organización, y de gran confianza al conocer de los directivos que lideran la administración, ya que el indicador ROE puede ser influenciado en gran manera debido al nivel de Utilidad neta que registre la institución, y esta sea confiable. Hoy en día, es complicado poder tomar decisiones de comprar acciones de una compañía en función del ROE, cuando desconocemos de la veracidad de sus estados financieros.

Palabras clave: ROE; utilidad neta; patrimonio; valor de mercado de la acciones; estados financieros.

\section{Abstract}

It is of great interest to know the profitability that we have when investing our capital in an organization, and of great confidence to know the managers who lead the administration, since the ROE indicator can be influenced in great way due to the level of Net Income that registers the institution, and this is reliable. Nowadays, it is difficult to make decisions to buy shares of a company based on the ROE, when we do not know the truth of its financial statements.

Keywords: ROE; net profit; heritage; market value of shares; financial statements.

\section{Resumo}

É muito interessante saber a rentabilidade temos de investir nosso capital em uma organização, e uma grande confiança de saber gestores que levam a administração, como o indicador ROE pode ser muito influenciado por causa do nível de lucro líquido para registar a instituição, e isso é confiável. Hoje, é difícil tomar decisões para comprar ações de uma empresa com base em ROE, quando não sabemos a veracidade das suas demonstrações financeiras.

Palavras chave: ROE; o lucro líquido; património; valor de mercado das ações; estados financeiros.

\section{Introducción}

En el contexto global, el campo de las finanzas desenvuelve un rol muy importante, debido a su gran influencia en el desarrollo y crecimiento económico de una nación, instituciones sin fines de lucro, organismos estatales, empresas privadas, entre otras. En consecuencia, las finanzas de cualquier índole deben ser manejada de forma consciente, objetiva, cognitiva y con la mayor 
experticia posible. La experiencia es muy importante en este campo, ya que permitirá tomar decisiones basadas, no sólo bajo un conocimiento técnico amplio, sino también en reflexiones sobre hechos pasados.

A nivel empresarial, las micro, pequeñas, medianas y grandes compañías buscan, siempre, generar beneficios y utilidad económica. Bajo esta premisa, los analistas financieros se enfocan diariamente por tomar las mejores decisiones tanto a corto como a largo plazo. Según Gitman \& Chad (2012):

"Las decisiones financieras a corto plazo se rigen por los mismos principios de administración financiera que las decisiones financieras a largo plazo; la diferencia reside solo en el tiempo: en el primer caso se trata de días, semanas y meses, en lugar de años. [...] si bien las decisiones financieras a largo plazo determinan, en última instancia, la capacidad de la empresa para incrementar al máximo la riqueza de los accionistas, podría no haber largo plazo si los gerentes fallan al tomar decisiones financieras efectivas a corto plazo”. (pág. 541)

Por lo general, los analistas se encuentran con disyuntivas financieras, tales como: endeudarse a corto o largo plazo, aumentar o disminuir el capital de trabajo, entre otras. Estas disyuntivas las resuelven con la experiencia que llevan en el campo de las finanzas, ya que ciertas herramientas, teóricamente correctas, suelen arrojar resultados no tan exactos comparados con la realidad. A su vez, el conocimiento técnico es relevante, ya que permite evaluar las decisiones tomadas por los gerentes o analistas financieros, haciendo uso de indicadores financieros.

Existen una gran cantidad de indicadores financieros, que permiten evaluar las condiciones financieras en que se encuentra la empresa en determinado momento; estos suelen clasificarse según la actividad económica que realice la compañía. Uno de los ratios más utilizados por los analistas financieros para medir el rendimiento que obtendría los accionistas por las inversiones realizadas, es el denominado Rendimiento sobre el Patrimonio o ROE. Aunque es muy utilizado, cabe destacar que es un indicador basado en valores contables y por lo general, son determinados subjetivamente. Entonces, ¿el Return on Equity (ROE), es un indicador financiero confiable para medir el rendimiento que obtendrían los accionistas de una empresa? De manera general, un indicador es el resultado del análisis de un conjunto de datos, por lo que no es aconsejable realizar análisis financieros de estos, ya que estos están compuestos por información resumida y a 
su vez, podría no mostrar detalles específicos que puedan confundir al analista/inversor en la toma de decisiones. El objetivo de la presente investigación es analizar minuciosamente la composición del ROE, y cualificar su funcionalidad para la toma de decisiones financieras.

El ROE, debido a su composición matemática y análisis financiero, no evidencia eficientemente el rendimiento que obtendría un inversor/accionista al inyectar liquidez por la compra de acciones de una empresa que cotiza en bolsa. A lo largo de la investigación, se resolverá dicha hipótesis.

\section{Metodología}

En cualquier campo de investigación, el aspecto metodológico es indispensable debido a su estrecha relación con la comprobación de la hipótesis propuesta y en efecto, del cumplimiento de los objetivos proporcionados anteriormente. Una correcta elección del diseño y tipo de investigación permitirá obtener resultados eficientes y apropiados para el estudio a realizarse.

El método principal que se aplicará a lo largo de esta investigación, es el método analítico deductivo. El principal objetivo de este método es la descomposición del "todo", lo que permite observar de forma detallada cada componente, en este caso del indicador financiero ROE. A su vez, se lo realizará de forma deductiva ya que se partirá de la ecuación principal y su funcionalidad, hasta la obtención de cada elemento perteneciente a dicha proposición.

A lo extenso de la investigación, se realizarán breves descripciones de los elementos intervinientes y su función en el indicador financiero. Debido a esto, el tipo de investigación descriptivo será el acorde para el cumplimiento de esta premisa. Sampieri, Fernández \& Baptista (2010), afirman que: "La investigación descriptiva busca especificar propiedades, características y rasgos importantes de cualquier fenómeno que se analice." (pág. 103) Además, la investigación documental o bibliográfica será indispensable para la realización de un mejor análisis y aporte académico.

\section{Discusión y resultado}

En primer lugar, hay que destacar que el Return on Equity (ROE), es un indicador financiero que relaciona el beneficio neto, obtenido en un periodo determinado, con el patrimonio que posee la empresa. Es decir, que la relación es netamente con valores contables, cuentas de resultados y balances, respectivamente. Algunos analistas y académicos consideran que el uso del ROE es 
muy importante para la toma de decisiones por parte de los accionistas, ya que esta mediría el rendimiento que obtendrían por inyectar liquidez a través de la compra de acciones.

Además, analistas financieros consideran que el ROE es un indicador que evidencia la rentabilidad para los accionista en aquellas empresas que no cotizan en bolsa, pero si dicha premisa fuera cierta, al menos este indicador sería un aproximado para la rentabilidad, y para los accionista en la empresas que cotizan en bolsa Fernández (2015).

Un indicador, es el resultado del análisis de un conjunto de datos, lo cual no permite realizar un exhaustivo análisis financiero debido a la información resumida que posee. Entonces, a manera general, un indicador no es una herramienta confiable, porque dentro de él pueden existir valores o detalles específicos tergiversados para la conveniencia de quien realiza el análisis. La composición matemática del ROE es la siguiente:

\section{Ecuación 1.}

$$
R O E=\frac{\text { Utilidad neta para accionistas }}{\text { Capital Contable Común (patrimonio })}
$$

Observando la ecuación 1, los dos componentes principales son: la utilidad neta para accionistas (Beneficio neto) y el capital contable común (patrimonio). El primer detalle importante que oculta este indicador es la presencia de la utilidad neta dentro del patrimonio, lo cual hace que el indicador se subestime y por ende las decisiones tomadas bajo el análisis de este indicador no serán las apropiadas. Un aspecto más general, es la presencia de valores contables, es decir proporcionan valores netamente estáticos por ende no considera la evolución en los próximos periodos de la empresa.

El caso Lehman Brothers, es una situación muy relevante al momento de contrastar los valores contables con la valorización de las acciones y rendimiento de los mismos; este banco mantenía en números azules cuentas contables, mientras que el valor de sus acciones iba en descenso, claro ejemplo de que la contabilidad de una empresa puede ser manipulada para intereses propios y ocultar información importante.

El valor de las acciones es de procedencia especulativa y no estática como lo brinda el indicador financiero ROE, debido a esto, las valoraciones de las empresas, existiendo excepciones, no son 
realizadas bajo el método contable sino bajo el método teóricamente correcto que es mediante el descuento del flujo (Fernández, 2015).

Para la creación de valor para los accionistas es necesario conocer cuatro conceptos muy importantes que permitirán realizar el cálculo de la rentabilidad para los accionistas, la rentabilidad que exigen los accionistas y por último obtener la creación de valor para los accionistas. Para llegar a la rentabilidad para los accionistas, es indispensable definir el aumento de la capitalización bursátil, el aumento de valor para accionistas.

La capitalización de una empresa que cotiza en el mercado bursátil, no es nada más que el valor de mercado, es decir el producto entre el precio de la acción y el número de acciones. El aumento de la capitalización bursátil en un año está determinado por la diferencia entre la capitalización final del año anterior y la capitalización final del año actual.

Ecuación 2.

\section{Aumento de Capitalización $=$ Capitalización $n_{1}-$ Capitalización $_{t-1}$}

Por otro lado, el aumento del valor para accionista considera la riqueza que se posee a final de cada año; es decir, el aumento está dado por la diferencia entre la riqueza al final del año 1 y la riqueza final del año 0 . Cabe recalcar que el aumento de capitalización no es el aumento del valor para accionista. Cuando se da un aumento de capitalización y no del valor para accionistas es debido a la presencia de ampliación de capital, en contraste, se da una disminución de capitalización pero el valor del accionista aumenta cuando la empresa paga dividendos, derechos.

Tabla 1. Cálculo del valor para accionistas

\section{Aumento del valor para accionistas}

= aumento de la capitalización de las acciones +

Dividendos pagados en el año -

Desembolso por ampliación de capital +

Otros pagos a los accionistas -

Conversión de obligaciones convertibles 
Fuente:Fernández(2015)

Elaboración Propia

La rentabilidad para los accionistas es la relación entre el aumento del valor de accionistas y la capitalización de la empresa al inicio del período. El cálculo se lo realiza de la siguiente forma:

Ecuación 3.

$$
\text { Rentabilidad para los accionistas }=\frac{\text { Aumento del valor de accionista }}{\text { Capitalización }}
$$

Es decir, que la rentabilidad para los accionistas está establecida por una relación inversamente proporcional a los cambios que se den en la capitalización de la empresa.

La rentabilidad exigida por los accionistas, es la rentabilidad que esperan los inversores a cambio de la inyección de liquidez mediante la adquisición de acciones. Esta rentabilidad debe ser mayor que la brindada por el Estado por los bonos a largo plazo. Además, debe tener en cuenta los riesgos sistemáticos, por ende la rentabilidad exigida por los accionistas deben considerar tanto la tasa libre de riesgo como la prima de riesgo de empresa.

Ecuación 4.

$$
K_{e}=\text { rentabilidad de bonos del Estado + prima de riesgo empresa }
$$

Y por último, ya definido las tres concepciones, calcular el valor creado por los accionistas es sólo la unificación de conceptos y fórmulas matemáticas. El cálculo se lo realiza bajo la siguiente fórmula:

Ecuación 5.

\section{Capitalización $\times\left(\right.$ Rentabilidad accionistas $\left.-K_{e}\right)$}

Siguiendo la lógica de esta fórmula, una empresa genera valor para los accionistas siempre y cuando la rentabilidad para los accionistas sea mayor que el costo de las acciones. 


\section{Conclusiones}

Cabe destacar que, basar la valorización de las acciones bajo valores contables es totalmente erróneo, debido a que estos, aparte de no tomar en cuenta la evolución de la empresa, no toma en cuenta externalidades como: caídas en el sector, pérdida de competitividad, crisis en la economía nacional, entre otros.

Es muy notorio que el valor de las acciones y por ende los rendimientos que estas brindan han sido influenciado siempre por los fenómenos que suceden en un momento determinado, por ejemplo: las burbujas especulativas.

El valor de las acciones es de procedencia especulativa y no estática como lo brinda el indicador financiero ROE, debido a esto las valoraciones de las empresas, existiendo excepciones, no son realizadas bajo el método contable sino bajo el método teóricamente correcto que es mediante el descuento del flujo.

\section{Referencias bibliográficas}

Fernández, P. (2000). El ROE no es la rentabilidad para los accionistas. En Creación de valor para los accionistas (págs. 21 - 30). New York: Gestión 2000.

Fernández, P. (2015). Cómo medir y gestionar la creación de valor. En Valoración de las Empresas. New York: Gestión 200.

Gitman, L., \& Chad, Z. (2012). Principios de Administración Financiera. México: Pearson.

Hernández Sampieri, R., Fernández Collado, C., \& Baptista Lucio, P. (2010). Metodología de la Investigación. México: McGraw-Hill Interoamericana.

Van Horne, J., \& Wachowics, J. (2010). Fundamentos de Administración Financiera. México: Pearson. 